\title{
Experiences from the Shiga toxin-producing Escherichia coli O104:H4 outbreak in Germany and research needs in the field, Berlin, 28-29 November 2011
}

STEC Workshop Reporting Group ${ }^{1}$

1. Members of the group and the corresponding author are listed at the end of the article

Citation style for this article:

STEC Workshop Reporting Group. Experiences from the Shiga toxin-producing Escherichia coli 0104:H4 outbreak in Germany and research needs in the field,

Berlin, 28-29 November 2011.

Euro Surveill. 2012;17(7):pii=20091. Available online: http://www.eurosurveillance.org/ViewArticle.aspx?Articleld=20091

Article published on 16 February 2012

This report presents the main findings from an international workshop on Shiga toxin-producing Escherichia coli (STEC), held on 28-29 November 2011, organised by the Robert Koch Institute [1]. The workshop assembled over 100 experts in clinical medicine, epidemiology, public health, microbiology, food safety, and environmental science from various countries.

\section{The 2011 outbreak}

From May to July 2011, Germany experienced an unprecedented outbreak of bloody diarrhoea and haemolytic uraemic syndrome (HUS) mostly among previously healthy adults caused by STEC serotype 0104: $\mathrm{H}_{4}$ [2-4]. Travellers from several countries were affected and an autochthonous outbreak caused by the same strain occurred in France [5]. Fenugreek sprouts were identified as the vehicle of infection [6].

Although the outbreak was controlled successfully, significant scientific challenges remain. To address these challenges, the workshop organised four working groups to discuss research needs in the following areas: (i) clinical issues, (ii) epidemiology, prevention and burden of disease, (iii) microbiology and pathogenesis and (iv) food safety, zoonotic and environmental aspects. The major research needs are presented below.

\section{Clinical issues}

As an important characteristic of the outbreak, many patients experienced severe clinical symptoms, and case fatality was substantial. Almost $50 \%$ of the HUS patients needed dialysis and a similar proportion developed neurologic symptoms [7]. Meanwhile, the vast majority of patients have completely recovered. No causal therapy for HUS exists, and pooled analysis of previous data showed no benefit of antibiotic treatment. In this outbreak, novel strategies for treating patients with HUS were applied (eg, antibody treatment with Eculizumab, immunoadsorption). The overall goal should be to optimise early diagnosis and care for patients with STEC-associated disease to reduce mortality and prevent organ damage.

Disease course and outcome: manifestations and predictors The natural disease course including the overall frequency and severity of the involvement of different organ systems should be investigated. Another aim is to study the risk factors and protective factors for the development of the different stages of STEC disease such as. bloody diarrhoea, HUS, neurological symptoms, recovery, and fatal outcome. This should include demographic and clinical characteristics, as well as genetic and environmental factors. Appropriate biomarkers (e.g. complement activation, chemokines, cytokines) indicating the risk of severe disease should be determined. Risk scores should be developed to identify and adequately treat patients at increased risk of HUS. The available clinical data could be used to develop an algorithm for standardised diagnostic procedures. The pathogenetic mechanisms underlying severe organ-specific lesions and recovery should be investigated (e.g. kidney, central nervous system).

Effectiveness and safety of treatment strategies This outbreak provides a unique opportunity to study the effects of antibiotics on the disease course. The effectiveness and safety of antibody treatment and immunoadsorption (short and long term outcomes) should be studied. Moreover, new therapeutic strategies to effectively remove Shiga toxin from the gastrointestinal tract once STEC has been diagnosed should be developed. Based on the scientific data obtained in this outbreak, recommendations for the future use of new and already existing therapeutic measures and international study protocols for treatment of STEC-HUS are warranted. 
Epidemiology, prevention and burden of disease

Research questions were collected and categorised according to high, moderate or low priority. Some areas may not belong to epidemiologic research in a stricter sense, but relate primarily to improving outbreak detection and control and STEC surveillance. The four topics outlined in the sections below were considered of utmost importance.

\section{Laboratory surveillance for outbreak detection}

Research should be targeted towards developing an optimal strategy for STEC surveillance. Diagnosis of all STEC is complex, but it is paramount to have a scheme allowing for timely assessment of the virulence profile of the causative agents and other characteristics (e.g. serotype) facilitating basic identification of clusters or of strains amenable for further subtyping (e.g. 0157). The usefulness of improved microbiological methods for primary detection of STEC and other diarrhoeagenic E. coli for clinical and public health purposes should be studied. Molecular subtyping is a powerful tool for detecting and investigating food-borne outbreaks, and its implementation is recommended. Research should identify the most useful typing methods and evaluate its cost-effectiveness.

\section{Product-tracing investigations}

as an epidemiological tool

The STEC 0104: $\mathrm{H}_{4}$ outbreak exemplified the value of product tracing investigations to identify suspected foods in outbreaks (e.g. by linking tracing information to disease clusters). Although successfully applied previously, the methodology is used infrequently thus far. Furthermore, it should be applied as an epidemiological tool for incriminating (or exonerating) suspected food items in outbreaks. It should be further developed and standardised as a tool in analytical epidemiological investigations. In this context it is necessary that legislation for public health or food safety allows for efficient usage of the methodology in food-borne outbreaks.

\section{Economic costs of the outbreak}

\section{/ Burden of illness}

This outbreak was unprecedented with respect to the number of HUS cases. Patient treatment is expensive (and long-term consequences are apprehended). Moreover, the outbreak led to costly trade-bans. Similarly, the burden of infectious intestinal diseases in general is substantial. Little is known about the overall burden of these infections, the contribution of the different pathogens to this burden, and the pathogen-specific risk factors. Estimating the costs of this outbreak and of infectious intestinal disease in general (e.g. disability adjusted life years, modelling) is pivotal to guide decision makers in rationally allocating financial resources for research and surveillance of infectious diseases. Population-based data on the pathogen-specific gastrointestinal disease burden are needed to improve targeted prevention and control measures.

\section{Clinical epidemiology}

The risk factors for the short-term and the long-term outcome in patient cohorts should be studied. Another research topic is the frequency and determinants of secondary transmission (households etc.) of STEC 0104: $\mathrm{H}_{4}$ and other serotypes.

\section{Microbiology and pathogenesis}

This outbreak vividly demonstrated the need to rapidly detect and molecularly characterise emerging STEC pathogens. Pathogenesis and evolution of STECmediated HUS are incompletely understood.

\section{Microbiological diagnosis}

Rapid and innovative methods facilitating early diagnosis of STEC (including emerging strains) should be established. Such approaches should combine molecular detection, culture and isolation of the pathogen and improve both outbreak detection and control and patient management. Methods to type and characterise such pathogens need to be improved. This requires the most advanced sequencing and data management capacities. It is a priority to implement molecular surveillance of STEC, including a central global database, analogous to PulseNet [8], containing typing and virulence data from human STEC isolates linked to STEC databases for animals, food and environment. Methods to detect viable but non-culturable STEC should be improved. It is important to optimise patient management via symptom-based approaches in order to accelerate diagnosis, implement best clinical and infection control practices, and to detect outbreaks as early as possible. Determining the duration of pathogen shedding by patients and quantifying the number of asymptomatic shedders is critical for epidemiological purposes. This should also include enteroaggregative E. coli (EAEC) and other diarrhoeagenic E. coli.

\section{Pathogenesis and evolution}

More evidence is needed on virulence and fitness factors of STEC, and on the mechanisms of their interaction with the host cells and their targets. In particular the roles of Shiga toxins and other toxins, serine proteases, and adhesins, iron uptake systems and growth-promoting factors expressed by these organisms should be delineated. Resistance of STEC to environmental stress, and the environmental factors that promote genetic changes in and spread of STEC virulence factors should be studied (role of phages, plasmids, and other mobile genetic elements). Infection models to study virulence and fitness under different conditions should be refined. Novel host and microbe targets for therapy have to be identified. Another research question relates to the protective host mechanisms including immune response and the role of commensal microbiota. The phylogenetic origin of STEC and their recent evolutionary emergence should be determined. The reservoirs, vectors and transmission 
of STEC and other diarrhoeagenic E. coli warrant further investigation.

\section{Antimicrobial therapy}

A major objective is to determine the effect of antimicrobials on stx-phage induction, Shiga toxin production, virulence and resistance gene transfer, and intestinal adherence (in vitro and in animal models). The mechanisms of transfer of antimicrobial resistance, for example extended-spectrum beta-lactamase, via plasmids in response to variable selective pressures should be delineated. The role of biofilm formation in STEC antimicrobial resistance and niche persistence during infection and in the environment should be studied, and the role of antimicrobial therapy in shortening bacterial shedding should be determined.

\section{Food safety, zoonotic and}

\section{environmental aspects}

Food safety

Optimised detection methods are needed for investigations of survival and multiplication of STEC 0104: $\mathrm{H}_{4}$ and other STEC in food matrices including fruits and vegetables. Research should be dedicated to the tracing of trading connections. Information systems providing real-time information from different market segments and incorporating the identification of risk factors as well as elements of predictive microbiology should be implemented. Risk profiling and ranking of combinations of foodstuffs and pathogens should be conducted (based on criteria such as microbiology, food processing and consumption, infectious dose, burden of disease). This will allow to identify the optimal sampling and control points along the food chain as well as the most efficient processing steps for decontamination based on hazard analysis and critical control points (HACCP). Decontamination methods for different types of foodstuff should be investigated. These should also take into consideration microbiological characteristics such as biofilm formation.

\section{Zoonotic aspects \\ Controlled infection experiments with various animal species may provide information about the potential of STEC $0104: \mathrm{H}_{4}$ to cross the species barrier from humans to animals and to establish itself in livestock, wild or pet animals. Studies in animals putatively exposed to STEC 0104: $\mathrm{H}_{4}$ during the outbreak or sub- sequently should elucidate whether the pathogen has already spread to animals and is maintained in animal populations.}

Reducing faecal STEC shedding and lowering its prevalence in livestock are promising strategies to protect humans from zoonotic STEC infection. Research is needed to understand host factors that predispose ruminants to STEC infection and shedding such as innate and acquired immunity, intestinal microbiota, genetic background and extrinsic factors such as livestock production systems. Innovative immunisation schemes as well as novel diets and pre- and probiotics against intestinal STEC colonisation in ruminants should be investigated.

\section{Environmental aspects}

The risks for products of animal and plant origin becoming contaminated by STEC originating from environmental sources such as soil, water, sewage systems, waste, human and animal shedding should be assessed. Laboratory studies and predictive microbiology may be used for the investigation of survival and multiplication of STEC in different microenvironments, also accounting for possible biofilm formation. Improved sampling and detection methods for environmental samples should be developed. The distribution of environmental STEC should be investigated along the food production chain as well as the horizontal transfer of virulence and antibiotic resistance genes between $E$. coli and other bacteria in these habitats. Research should address the issue of selective pressure by environmental or management factors contributing to the development of niches facilitating the survival or multiplication of STEC and the emergence of new $E$. coli seropathotypes. Decontamination methods for waste water, surface waters and production water such as water used for the irrigation of plants should be assessed.

\section{Conclusions}

This massive outbreak had substantial international implications. It exemplified that the landscape of food-borne infections is in flux, that multi-national outbreaks are a reality and that they can occur everywhere, irrespective of food safety standards.

The workshop showed that urgent and challenging research needs exist in the field of STEC and other diarrhoeagenic $E$. coli, and as far as burden of illness is concerned also of other food-borne pathogens. The scientific questions identified need to be further prioritised and strategies to address them should be developed. The workshop delineated interfaces between the working groups. Among those, the most notable are (i) the need to further develop diagnostic methods and to integrate molecular typing into routine surveillance, (ii) to study the pathogenesis, clinical course and new treatment options, (iii) to make use of systematic food tracing data as epidemiological tools, and (iv) to study pathogen evolution among the human host, the environment, and in animal reservoirs.

These research needs must be addressed soon in order to better equip clinicians, microbiologists, public health and food safety authorities for the early detection and efficient control of food-borne outbreaks and to prevent similar events. As it is an obligation of the scientific community to investigate these research questions it is also in the responsibility of national and international funding bodies to fund the respective research programmes. 
The workshop was funded by the Ministry of Health, Germany.

Members of the STEC Workshop Reporting Group in alphabetical order:

Corresponding author: K Stark (starkk@rki.de), Robert Koch Institute, Berlin, Germany, R Bauerfeind, Institute for Hygiene and Infectious Diseases of Animals, Justus Liebig University Giessen, Giessen, Germany, H Bernard, Robert Koch Institute, Berlin, Germany, T Eckmanns, Robert Koch Institute, Berlin, Germany, S Ethelberg, Statens Serum Institut, Copenhagen, Denmark, A Flieger, Robert Koch Institute, Berlin, Germany, J Giesecke, European Centre for Disease Prevention and Control (ECDC), Stockholm, Sweden, M Greiner, Federal Institute of Risk Assessment, Berlin, Germany, H Karch, Institute of Hygiene, University Münster, Münster, Germany, G Krause, Robert Koch Institute, Berlin, Germany, M Mielke, Robert Koch Institute, Berlin, Germany , SJ O'Brien, Institute of Infection and Global Health, National Centre for Zoonosis Research, University of Liverpool, United Kingdom, M Pulz, State Authority for Public Health Lower Saxony, Hannover, Germany, F Scheutz, Statens Serum Institut, Copenhagen, Denmark, A Schielke, Robert Koch Institute, Berlin, Germany, RA Stahl, Department of Medicine, Division of Nephrology, University Hospital Hamburg-Eppendorf, Hamburg, Germany, J Takkinen, European Centre for Disease Prevention and Control (ECDC), Stockholm, Sweden, PI Tarr, Department of Pediatrics, Division of Gastroenterology and Nutrition, Washington University in St. Louis, United States, RV Tauxe, Centers for Disease Control and Prevention (CDC), Atlanta, United States, D Werber, Robert Koch Institute, Berlin, Germany.

\section{References}

1. International Workshop 'Experiences from the STEC 0104: $\mathrm{H}_{4}$ outbreak in Germany and research needs for STEC', 28-29 November 2011, Berlin, Germany.

2. Frank C, Werber D, Cramer JP, Askar M, Faber M, an der Heiden $M$, et al. Epidemic profile of Shiga-toxin-producing

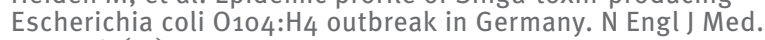
2011;365(19):1771-80.

3. Frank C, Faber MS, Askar M, Bernard H, Fruth A, Gilsdorf A, et al. Large and ongoing outbreak of haemolytic uraemic syndrome, Germany, May 2011. Euro Surveill, 2011:16(21). pii: 19878. Available from: http://www.eurosurveillance.org/ ViewArticle.aspx?Articleld $=19878$

4. Bielaszewska M, Mellmann A, Zhang W, Köck R, Fruth A, Bauwens A, et al. Characterisation of the Escherichia coli strain associated with an outbreak of haemolytic uraemic syndrome in Germany, 2011: a microbiological study. Lancet Infect Dis. 2011;11(9):671-6.

5. Gault G, Weill FX, Mariani-Kurkdjian P, Jourdan-da Silva N, King $\mathrm{L}$, Aldabe B, et al. Outbreak of haemolytic uraemic syndrome and bloody diarrhoea due to Escherichia coli $0104: \mathrm{H}_{4}$, southwest France, June 2011. Euro Surveill. 2011;16(26). pii: 19905. Available from: http://www.eurosurveillance.org/ViewArticle. aspx?Articleld $=19905$

6. Buchholz U, Bernard H, Werber D, Böhmer MM, Remschmidt C, Wilking H, et al. German outbreak of Escherichia coli $0_{104}: \mathrm{H}_{4}$ associated with sprouts. N Engl J Med. 2011;365(19):1763-70.

7. Stahl RA. Eculizumab in STEC-HUS. Presented at the 44 th Meeting of the American Society of Nephrology, November 8-13, 2011, Philadelphia, USA.

8. Swaminathan B, Barrett TJ, Hunter SB, Tauxe RV; CDC PulseNet Task Force. PulseNet: the molecular subtyping network for foodborne bacterial disease surveillance, United States. Emerg Infect Dis. 2001;7(3):382-9. 